Supplementary information for

\title{
Multiscale assay of unlabeled neurite dynamics using phase imaging with computational specificity (PICS)
}

Author List: Mikhail E. Kandel ${ }^{1,2} \neq$, Eunjae Kim ${ }^{1,2} \neq$, Young Jae Lee $e^{1,3}$, Gregory Tracy ${ }^{5}$, Hee Jung Chung $^{3,5}$, Gabriel Popescu ${ }^{1,2,4^{*}}$

1. Beckman Institute, University of Illinois at Urbana-Champaign, Urbana, IL 61801, USA

2. Department of Electrical and Computer Engineering, University of Illinois at UrbanaChampaign, Urbana, IL 61801, USA

3. Neuroscience Program, University of Illinois at Urbana-Champaign, Urbana, IL 61801, USA

4. Department of Bioengineering, University of Illinois at Urbana-Champaign, Urbana, IL 61801, USA

5. Department of Molecular Integrated Physiology, University of Illinois at Urbana Champaign, Urbana, IL 61801, USA

† Equal contributions 


\section{Supplementary Note 1: Gradient light interference microscopy}

a

GLIM Module

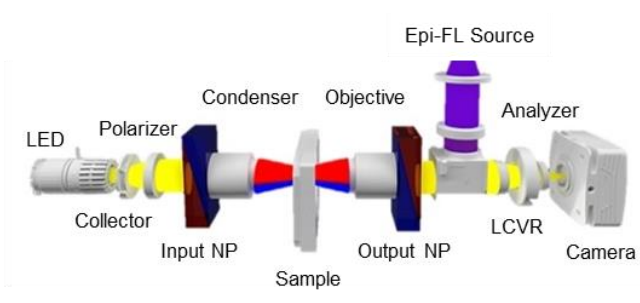

C

Phase and Amplitude Response
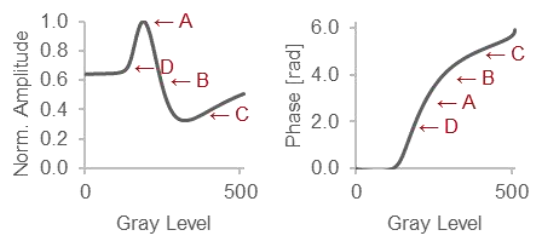

e

Hilbert Integration

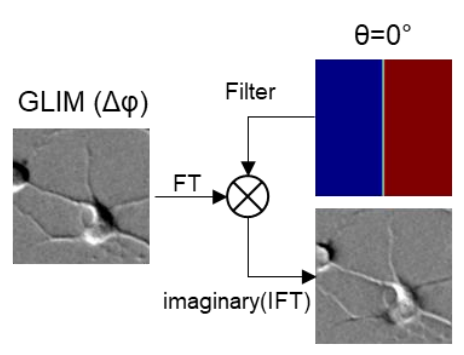

b

LCVR Gray Level Scan
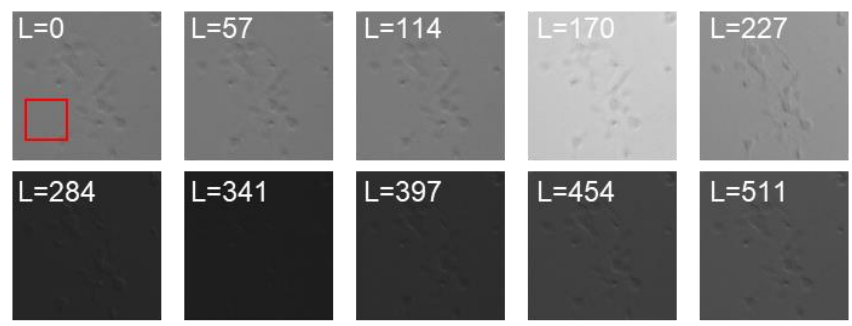

d

\section{Flatness Correction}
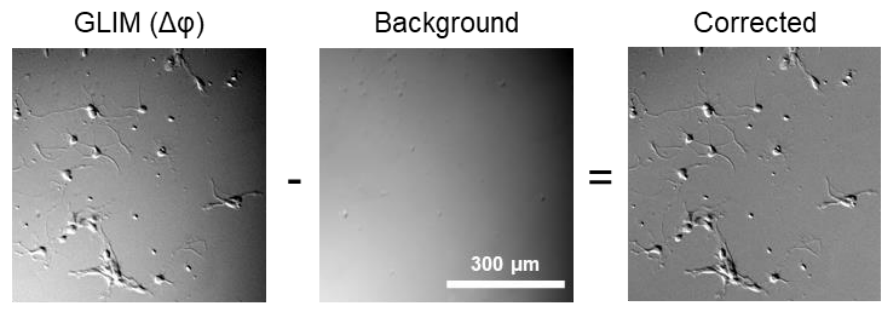

$-0.3$

$+0.3$

Supplementary Figure 1. Design and calibration of the GLIM module. a, Following the layout for DIC, the GLIM light path uses an input polarizer to introduce two beams that traverse the sample at laterally offset positions. These beams are combined in space by the output polarizer with the interference between the two polarizations controlled by a liquid crystal variable retarder mounted before the camera. b, To establish the relationship between the voltage on the modulator and the imparted phase shift, we perform a calibration procedure where "gray levels" with increasing phase shift are written to the modulator. c, Taking the average of these values over a simple-free region produces an amplitude modulation curve. Hilbert transform of which is the instantaneous phase associated with the modulator shift. d, To correct for background nonuniformities we perform background subtraction by removing an image corresponding to the 
average phase map acquired during the experiment. e, To integrate the phase we use a Hilbert transform where the Fourier transform of the GLIM image is multiplied by a filter corresponding to DIC shear direction. The imaginary part of the inverse transform contains the integrated phase. To determine the angle we evaluate all possible candidates (variations of $\phi$ shown) and select the one where all cellular structures introduce a positive phase shift $\left(\theta=315^{\circ}\right.$ in this case).

Gradient light interference microscopy (GLIM) is a quantitative phase imaging technique that is constructed as an add-on module to a conventional DIC microscope ${ }^{1}$. In this work, we use a liquid crystal variable retarder-based design first presented in ${ }^{2}$. GLIM is particularly well suited to reproduce turbid structures but also benefits from a relatively unobstructed fluorescent light path.

As shown in Supporting Fig. 1a, the GLIM light path begins with an incoherent light source. In this work, we use a red LED centered at $623 \mathrm{~nm}$ at the lowest power setting (Thorlabs, SOLIS623C). The red color helps avoid harmful UV radiation and the $\sim 50 \mathrm{~nm}$ bandwidth spectrum improves modulator contrast compared to a white light source. For imaging, we used a DIC microscope, which splits light into two beams that traverse the sample at slightly offset positions. In our implementation, we used an Axio Observer Z1 (Zeiss) equipped with an automated stage and mini-incubator. All imaging was performed on a 20x/0.8 objective with a fully open condenser (DICII/0.55). To obtain the quantitative phase information, we remove the output polarizer (in the filter cube) and place a liquid crystal variable retarder (LCVR, LVR-100-IR, Meadowlark) and polarizer immediately before the camera.

The LCVR is used to introduce a controlled phase shift between interfering beams in DIC which can then be used to recover the phase information. To establish the relationship between the gray-level and the phase shift we perform a calibration procedure where we acquire a sequence 
of 512 increasing voltages, conventionally referred to as "gray levels". In general, the calibration should be run for each light source, and this sequence resembles what would occur when rotating the de Sénarmont prism (Supporting Fig. 1b). To determine the relationship between gray-level and effective phase shift, we use a sample-free portion of the image and project the spatial average value as a function of gray-level (Supporting Fig. 1c). The instantaneous phase is recovered by the Hilbert transform of the normalized amplitude signal which results in the imaginary portion of the complex analytic signal, the argument of which is the phase. To obtain the final reconstruction formula we take the largest peak ("A") and find phase shifts that are $90^{\circ}, 180^{\circ}, 270^{\circ}$ from that point. To account for discretization errors we use a phase reconstruction formula that recovers the phase when points that are slightly off from $90^{\circ}$ intervals $^{2}$.

As the GLIM image is a measurement of the phase between DIC beams, it resembles a derivative of the scattering potential rather than the actual potential. To recover the phase shift associated with the object we remove the artificial slant-like background and divide by the previously measured DIC shear distance $(0.3 \mu \mathrm{m})$. In this work, we obtain a background image by averaging together all images in the time-lapse sequence (Supporting Fig. 1d), and for real-time imaging when setting up the experiment, we used Fourier filtering ${ }^{3}$.

Numerical integration is performed using a Hilbert transform, which is good at preserving highfrequency information while avoiding the need to measure an impulse response or use a regularizer. Comparing the Hilbert transform to more formal integration methods, we note that the principle distortion is in low-frequencies that lay outside GLIM's frequency response ${ }^{4}$. To determine the angle of integration precisely we use an exhaustive approach where we generate 360 images corresponding to different integration angles (Supporting Fig. 1e). The correct angle 
of integrations can be visually identified by choosing an image where all cellular structures appear as positive phase shifts (315 degrees in this case). In principle, it should be possible to read the angle from the frequency spectra or simply determine the shear angle from the construction of the instrument. 


\section{Supplementary Note 2: Cell-culture and immunostaining}

All procedures involving animals were reviewed and approved by the Institutional Animal Care and Use Committee at the University of Illinois Urbana-Champaign and conducted per the guidelines of the U.S National Institute of Health (NIH). Primary dissociated hippocampal neurons were prepared from the hippocampi which were dissected from Sprague-Dawley rat embryos at embryonic day 18 as described ${ }^{5}$ and plated onto a glass-bottom multiwell plate (Cellvis, P06-20-1.5-N) that was functionalized with poly-D-lysine (0.1 mg/ml; Sigma-Aldrich). Hippocampal neurons were initially incubated with a plating medium containing $86.55 \%$ MEM Eagle's with Earle’s BSS (Lonza), 10\% Fetal Bovine Serum (re-filtered, heat-inactivated; ThermoFisher), $0.45 \%$ of $20 \%$ (wt./vol.) glucose, 1x 100 mM sodium pyruvate (100x; SigmaAldrich), 1x 200 mM glutamine (100x; Sigma-Aldrich), and 1x Penicillin/ Streptomycin (100x; Sigma-Aldrich) to be attached to the surface of the well plates. Neurons were plated at 300 cells $/ \mathrm{mm}^{2}$, although the achieved density was calculated on a per-tile basis using image analysis. After three hours of incubation $\left(37^{\circ} \mathrm{C}\right.$ and $\left.5 \% \mathrm{CO}_{2}\right)$, the plating media was aspirated and replaced with maintenance media containing Neurobasal growth medium (ThermoFisher) supplemented with B-27 (ThermoFisher), 1\% $200 \mathrm{mM}$ glutamine (ThermoFisher), and 1\% penicillin/streptomycin (ThermoFisher) at $37^{\circ} \mathrm{C}$, in the presence of $5 \% \mathrm{CO}_{2}$. Hippocampal neurons were maintained for three days before imaging.

Timelapse microscopy was performed using the plate reader instrumentation developed in ${ }^{4}$. Imaging was performed on three wells with a $10 \times 10$ mosaic performed every 50 minutes. To avoid altering the polarization in DIC we used a glass top for imaging (Cellvis, L001). At the end of the experiment, cells were stained and imaged using co-localized fluorescence and GLIM imaging. 
In this study, antibodies for Tau (Abcam, ab80579) and MAP2 (Abcam ab32454) were used for immunostaining of axons and dendrites, respectively, following the established protocol from the ThermoFisher and $\mathrm{Abcam}^{6}$. In brief, neurons were fixed with freshly prepared 4\% paraformaldehyde (PFA) for 15 minutes following $0.5 \%$ Triton-X for 10 minutes and $2 \%$ BSA for 30 minutes incubation. Hippocampal neurons were incubated for 8 hours at $4^{\circ} \mathrm{C}$ with antiTau antibodies that were diluted to 1:250 in 1\% bovine serum albumin (BSA, ThermoFisher). After washing, neurons were exposed for 8 hours at $4{ }^{\circ} \mathrm{C}$ to goat anti-mouse secondary antibody (Abcam, ab205719) which was diluted to 1:500 in 1\% BSA. Hippocampal neurons were then incubated in anti-MAP2 antibody (1:500 dilution) in 1\% BSA for 8 hours, followed by goat antirabbit secondary antibody (Abcam, ab205718, 1:1000 dilution) in 1\% BSA for 8 hours at $4^{\circ} \mathrm{C}$. We found that the fluorescent signal covered roughly $7 \%$ of the histogram $(\sim 150$ counts from 2047 available). To improve this dynamic range, and we used a combination of 2 x 2 binning and 10 frames averaging for each fluorescence channel (Photometrics, BSI Prime). This fluorescence acquisition is roughly 70x slower than the time to acquire the GLIM image. 


\section{Supplementary Note 3: Semantic Segmentation Accuracy}

To evaluate the accuracy of our semantic segmentation we compare the actual and imputed fluorescent signals that are acquired at the end of the experiment and constitute the training corpus for deep learning. Thus, we generate semantic maps from the actual and imputed signals following the PCA method shown in the main manuscript Fig. 2. Supplementary Table 1, shows the performance in terms of sensitivity and specificity for each category along with two other well-known segmentation metrics: Dice index and Jaccard index ${ }^{7}$. The metrics are calculated for each field-of-view in the test dataset.

Supplementary Table 1: Semantic Segmentation Performance

\begin{tabular}{|c|c|c|}
\hline & Neurite & Nucleus \\
\hline Sensitivity & $80.76 \%( \pm 4.42 \%)$ & $83.22 \%( \pm 4.51 \%)$ \\
\hline Specificity & $96.73 \%( \pm 0.91 \%)$ & $99.85 \%( \pm 0.05 \%)$ \\
\hline Dice Index & $70.27 \%( \pm 5.16 \%)$ & $75.27 \%( \pm 3.59 \%)$ \\
\hline Jaccard Index & $54.40 \%( \pm 5.87 \%)$ & $60.47 \%( \pm 4.51 \%)$ \\
\hline
\end{tabular}




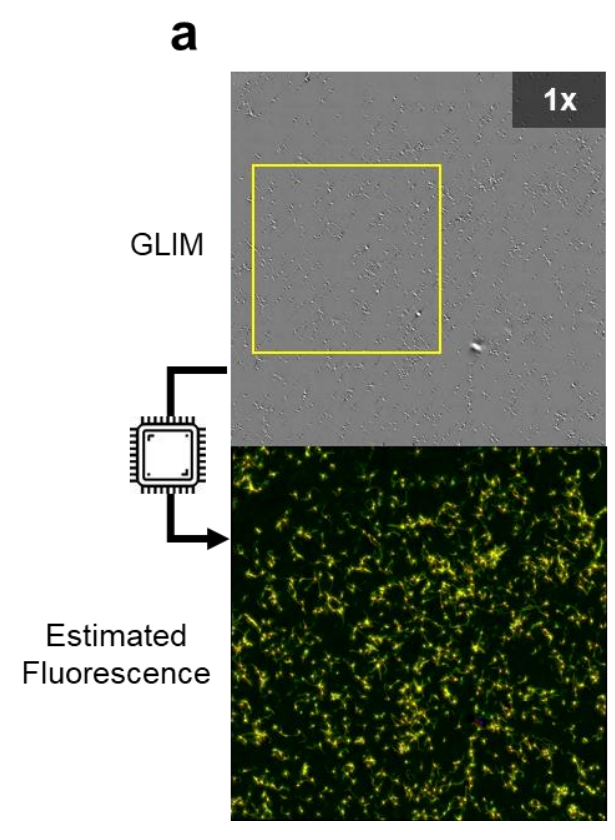

b

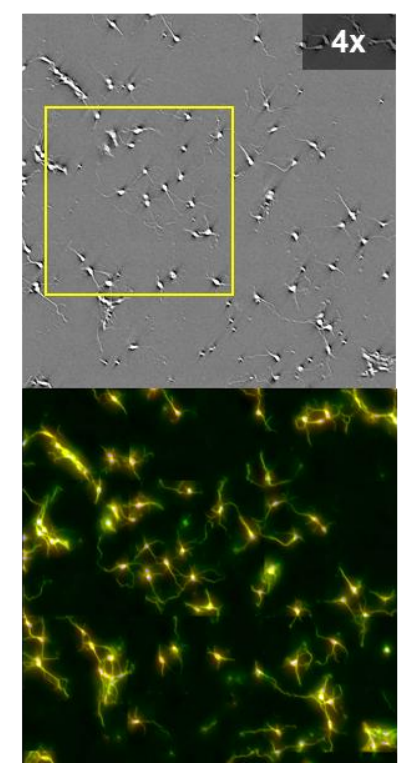

c

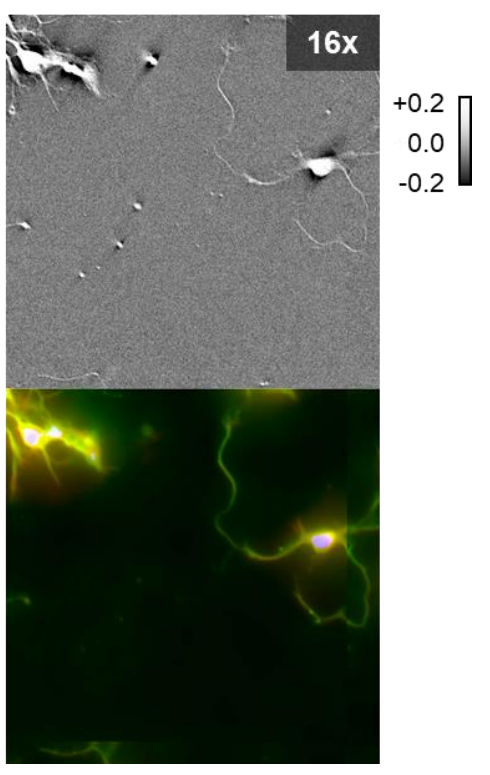

Supplementary Figure 2. Cellular level detail in GLIM and PICS. a-c Representative field of view shown alongside the estimated fluorescent signal at increasing zoom levels $(20 \mathrm{x} / 0.8)$.
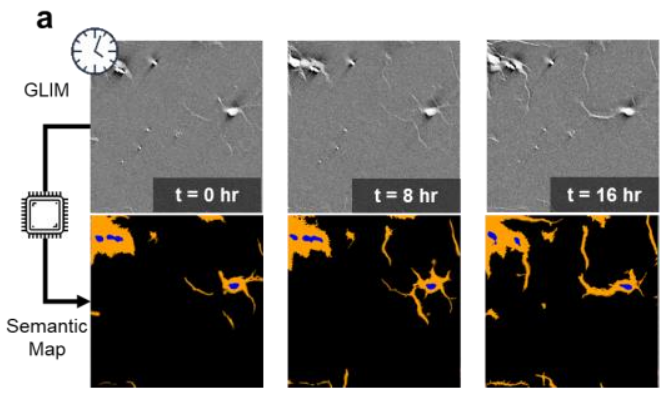

b
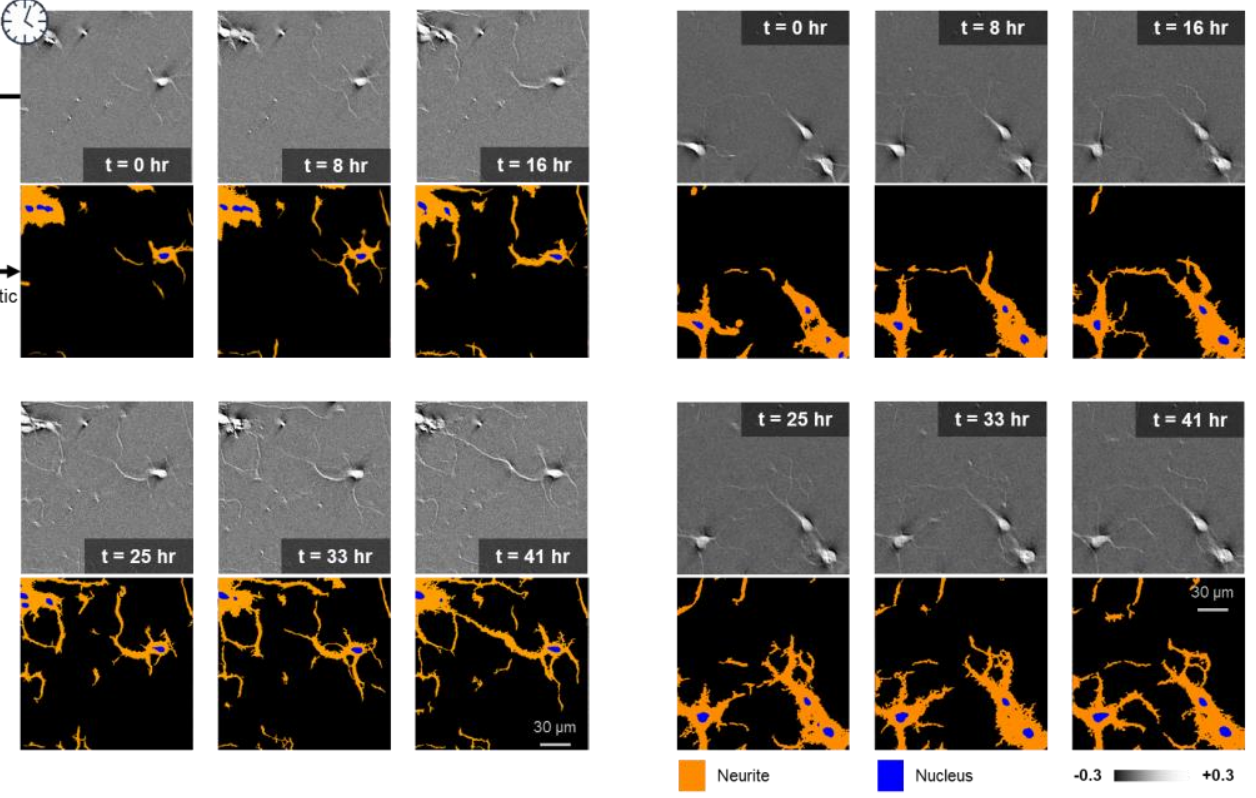

Supplementary Figure 3. Semantic segmentation and time-lapse development of the neuronal arbor. Two representative fields of view along with associated semantic segmentation $\operatorname{map}(20 \mathrm{x} / 0.8)$. 


\section{Supplementary Note 4: Dispersion-Relation Phase Spectroscopy for Mass Transport Analysis}

Cellular mass transport can be analyzed by tracking moving objects or by analyzing spectral fluctuations in the image. We opt for the latter as the neuronal arbor forms a dense network that approaches a continuous dynamic system. In this work, we propose to use dispersion-relation phase spectroscopy (DPS) ${ }^{8}$, which interprets the mass transport under the diffusion-advection equation. This method of analysis transforms a time-lapse image sequence into an estimate of the temporal bandwidth at each spatial mode, which can be used to calculate the diffusion/advection coefficients. The steps for this procedure are shown in Supplementary Fig. 4.

Following the derivation in ${ }^{9}$, we assume that the inhomogeneous mass density (dry mass or Tau protein) within a semantic label, $\rho(\mathbf{q}, t)$, follows the diffusion-advection equation,

$$
\left(-D \mathbf{q}^{2}+i \mathbf{q} \cdot \mathbf{v}-\frac{d}{d t}\right) \rho(\mathbf{q}, t)=0
$$

Where $D$ is the diffusion coefficient, and $\mathbf{v}$ is the directed velocity of the particles. We can write the velocity-averaged density as 9

$$
\langle\rho(\mathbf{q}, \tau)\rangle_{v}=\exp \left(i \mathbf{q} \cdot \mathbf{v}_{o} \tau\right) \exp \left(-\left[D \mathbf{q}^{2}+\Delta \mathbf{v} \cdot \mathbf{q}\right] \tau\right)
$$

where \langle\rangle$_{v}$ indicates ensemble averaging over the velocity distribution, $\mathbf{v}_{o}$ is the mean velocity, and $\Delta \mathbf{v}$ represents the variance of the distribution of velocities. The mean velocity, $\mathbf{v}_{o}$, is typically negligible, as mass transport is equally probably in both directions along a given line. Accordingly, the decay time at each spatial frequency, $\Gamma(\mathbf{q})$, characterizes the behavior of the sample, as 


$$
\Gamma(\mathbf{q})=D \mathbf{q}^{2}+\Delta \mathbf{v} \cdot \mathbf{q}
$$

To compute $\Gamma(\mathbf{q})$ efficiently, we evaluate it as the standard deviation of the temporal power spectrum. We note that while, in general, the second moment of a Lorentzian (Fourier transform of an exponential) is not defined, for these experiments we use finite imaging intervals which makes our integrals converge. For the zero-mean power spectra of interest here, the variance is computed as,

$$
\Gamma^{2}(\mathbf{q})=\frac{\int \omega^{2}|\rho(\mathbf{q}, \omega)|^{2} d \omega}{\int|\rho(\mathbf{q}, \omega)|^{2} d \omega}=\frac{\int\left|\frac{d \rho(\mathbf{q}, t)}{d t}\right|^{2} d t}{\int|\rho(\mathbf{q}, t)|^{2} d t}
$$

where $\rho(\mathbf{q}, \omega)$ is the Fourier transform of $\rho(\mathbf{q}, t)$ in time. Note that the differentiation property of the Fourier transform allows us to compute $\Gamma(\mathbf{q})$ directly in the time domain very quickly ${ }^{10}$. By curve fitting using the measured values of $\Gamma(\mathbf{q})$, it is possible to estimate $D$ and $\Delta \mathbf{v}$ for particular spatial frequency ranges. The isotropic intracellular transport is obtained by performing a radial average over $\Gamma(\mathbf{q})$. In this work, we develop a high-quality procedure for radial average that involves first mapping each point in $\Gamma$ to its radial coordinate $\|\mathbf{q}\|$ followed by an interpolation step (See Supplementary Fig. 4, Step 4: Radial Averaging).

As imaging was performed in 50-minute increments, we noticed that diffusive motion was negligible, i.e., the quadratic $\mathbf{q}^{2}$ term was small. This makes the curve-fitting a straightforward linear fit. These operations were performed in an automated fashion using a script written in MATLAB. 
For a more intuitive representation of the data, intervals of $q$ are converted into direct-space scales using the following relationship,

$$
\lambda=\frac{2 \pi}{q}
$$


a

Step 1: Data Acquisition

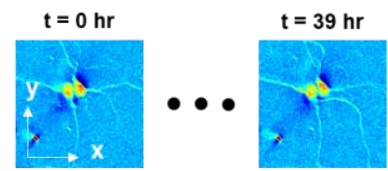

C

Step 3: Spectral Representation

$\mathrm{t}=0 \mathrm{hr}$

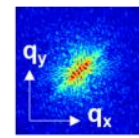

e

Step 5: Parameter Extraction

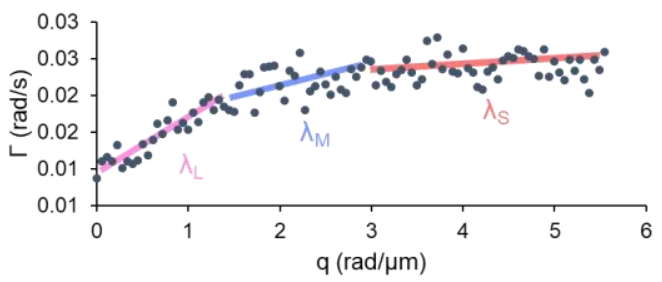

b

Step 2: Semantic Segmentation

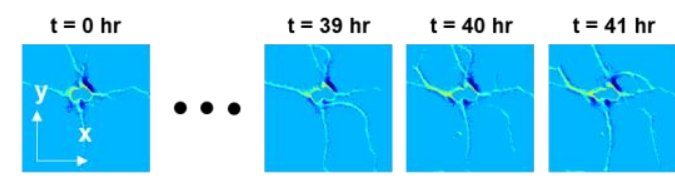

d

Step 4: Radial Averaging

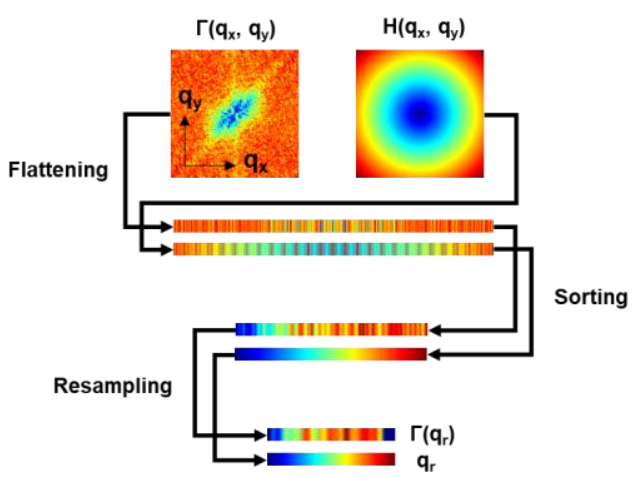

Supplementary Figure 4. Processing for dispersion-relation phase spectroscopy (DPS). a, The DPS procedure estimates the spread of the advection coefficients from a series of phase (shown) or fluorescent measurements. b, To apply the semantic segmentation to the phase image, we zero all values outside the labeled regions. c, Next, we take the Fourier transform of the sequence on a per tile basis. As expected, the resolution limit $\left(\mathrm{q}_{\max }\right)$ appears as a disk in the frequency domain. d, Next we estimate the variance of the autocorrelation using a finite difference scheme to obtain $\Gamma\left(\mathrm{q}_{\mathrm{x}}, \mathrm{q}_{\mathrm{y}}\right)$. As cellular transport is assumed to be anisotropic we perform a radial average by indexing $\Gamma$ with $q$ [the modulus of $\mathbf{q}=\left(\mathrm{q}_{\mathrm{x}}, \mathrm{q}_{\mathrm{y}}\right)$ ] followed by a resampling step to reduce the radially sorted data from $\sim 1$ megapixels to a more manageable 1,000 samples. f, Finally, we perform a linear curve fit over the ranges corresponding to "small" $\left(\lambda_{S}\right)$, "medium" $\left(\lambda_{M}\right)$, and "large" $\left(\lambda_{L}\right)$ scale features. 


\section{Video 1}

GLIM and PICS estimated fluorescence for Tau/MAP2/DAPI for the first hippocampal culture. Imaging was performed for 41 hours at (20x/0.8).

\section{Video 2}

GLIM and PICS estimated fluorescence for Tau/MAP2/DAPI for the second hippocampal culture. Imaging was performed for 41 hours at (20x/0.8).

\section{Video 3}

GLIM and PICS estimated fluorescence for Tau/MAP2/DAPI for the third hippocampal culture. Imaging was performed for 41 hours at (20x/0.8). 


\section{References and Notes:}

1. Nguyen, T. H.; Kandel, M. E.; Rubessa, M.; Wheeler, M. B.; Popescu, G., Gradient light interference microscopy for 3D imaging of unlabeled specimens. Nat Commun 2017, 8 (1), 210.

2. Kandel, M. E.; Hu, C.; Naseri Kouzehgarani, G.; Min, E.; Sullivan, K. M.; Kong, H.; Li, J. M.; Robson, D. N.; Gillette, M. U.; Best-Popescu, C.; Popescu, G., Epi-illumination gradient light interference microscopy for imaging opaque structures. Nat Commun 2019, 10 (1), 4691.

3. Kandel, M. E.; Teng, K. W.; Selvin, P. R.; Popescu, G., Label-free imaging of single microtubule dynamics using spatial light interference microscopy. ACS nano 2017, 11 (1), 647655.

4. Kandel, M. E.; He, Y. R.; Lee, Y. J.; Chen, T. H.; Sullivan, K. M.; Aydin, O.; Saif, M. T. A.; Kong, H.; Sobh, N.; Popescu, G., Phase imaging with computational specificity (PICS) for measuring dry mass changes in sub-cellular compartments. Nat Commun 2020, 11 (1), 6256.

5. Zhang, J.; Kim, E. C.; Chen, C.; Procko, E.; Pant, S.; Lam, K.; Patel, J.; Choi, R.; Hong, M.; Joshi, D.; Bolton, E.; Tajkhorshid, E.; Chung, H. J., Identifying mutation hotspots reveals pathogenetic mechanisms of KCNQ2 epileptic encephalopathy. Sci Rep 2020, 10 (1), 4756.

6. Abcam Immunocytochemistry and immunofluorescence protocol https://www.abcam.com/protocols/immunocytochemistry-immunofluorescence-protocol. 7. Kayalibay, B.; Jensen, G.; van der Smagt, P., CNN-based segmentation of medical imaging data. arXiv preprint arXiv:1701.03056 2017.

8. Wang, R.; Wang, Z.; Millet, L.; Gillette, M. U.; Levine, A. J.; Popescu, G., Dispersionrelation phase spectroscopy of intracellular transport. Opt Express 2011, 19 (21), 20571-9.

9. Kandel, M. E.; Sridharan, S.; Liang, J.; Luo, Z.; Han, K.; Macias, V.; Shah, A.; Patel, R.; Tangella, K.; Kajdacsy-Balla, A.; Guzman, G.; Popescu, G., Label-free tissue scanner for colorectal cancer screening. J Biomed Opt 2017, 22 (6), 66016.

10. Popescu, G., Principles of Biophotonics, Volume 1. In Linear systems and the Fourier transform in optics, IOP Publishing: 2018. 